\title{
DNA methylation mediates the effect of cocaine use on HIV severity
}

\author{
Chang Shu ${ }^{1,2}$, Amy C. Justice ${ }^{2,3}$, Xinyu Zhang ${ }^{1,2}$, Zuoheng Wang ${ }^{4}$, Dana B. Hancock ${ }^{5}$, Eric O. Johnson ${ }^{5,6}$ and
} $\mathrm{Ke} X \mathrm{u}^{1,2^{*}}$ (i)

\begin{abstract}
Background: Cocaine use accelerates human immunodeficiency virus (HIV) progression and worsens HIV outcomes. We assessed whether DNA methylation in blood mediates the association between cocaine use and HIV severity in a veteran population.

Methods: We analyzed 1435 HIV-positive participants from the Veterans Aging Cohort Study Biomarker Cohort (VACS-BC). HIV severity was measured by the Veteran Aging Cohort Study (VACS) index. We assessed the effect of cocaine use on VACS index and mortality among the HIV-positive participants. We selected candidate mediators that were associated with both persistent cocaine use and VACS index by epigenome-wide association (EWA) scans at a liberal $p$ value cutoff of 0.001 . Mediation analysis of the candidate $\mathrm{CpG}$ sites between cocaine's effect and the VACS index was conducted, and the joint mediation effect of multiple CpGs was estimated. A two-step epigenetic Mendelian randomization (MR) analysis was conducted as validation.

Results: More frequent cocaine use was significantly associated with a higher VACS index $(\beta=1.00, p=2.7 \mathrm{E}-04)$, and cocaine use increased the risk of 10-year mortality (hazard ratio $=1.10, p=0.011$ ) with adjustment for confounding factors. Fifteen candidate mediator CpGs were selected from the EWA scan. Twelve of these CpGs showed significant mediation effects, with each explaining $11.3-29.5 \%$ of the variation. The mediation effects for 3 of the $12 \mathrm{CpGs}$ were validated by the two-step epigenetic MR analysis. The joint mediation effect of the $12 \mathrm{CpGs}$ accounted for $47.2 \%$ of cocaine's effect on HIV severity. Genes harboring these $12 \mathrm{CpGs}$ are involved in the antiviral response (IFIT3, IFITM1, NLRC5, PLSCR1, PARP9) and HIV progression (CX3CR1, MX1).
\end{abstract}

Conclusions: We identified 12 DNA methylation $C p G$ sites that appear to play a mediation role in the association between cocaine use and HIV severity.

Keywords: Cocaine use, HIV severity, Mortality, DNA methylation, Mediation effect

\footnotetext{
* Correspondence: ke.xu@yale.edu

${ }^{1}$ Department of Psychiatry, Yale School of Medicine, New Haven, CT, USA

${ }^{2}$ Connecticut Veteran Healthcare System, West Haven, CT, USA

Full list of author information is available at the end of the article
}

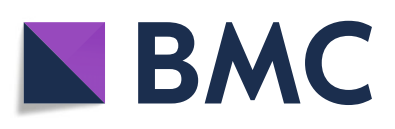

(c) The Author(s). 2020 Open Access This article is licensed under a Creative Commons Attribution 4.0 International License, which permits use, sharing, adaptation, distribution and reproduction in any medium or format, as long as you give appropriate credit to the original author(s) and the source, provide a link to the Creative Commons licence, and indicate if changes were made. The images or other third party material in this article are included in the article's Creative Commons licence, unless indicated otherwise in a credit line to the material. If material is not included in the article's Creative Commons licence and your intended use is not permitted by statutory regulation or exceeds the permitted use, you will need to obtain permission directly from the copyright holder. To view a copy of this licence, visit http://creativecommons.org/licenses/by/4.0/ The Creative Commons Public Domain Dedication waiver (http://creativecommons.org/publicdomain/zero/1.0/) applies to the data made available in this article, unless otherwise stated in a credit line to the data. 


\section{Introduction}

Cocaine use is common among persons with chronic human immunodeficiency virus (HIV) infection, with prevalence estimates for current or recent use ranging from 5 to $30 \%$ [1-6], compared with $2 \%$ in the US general population [7]. Previous studies have shown that cocaine use accelerated HIV progression [8-11]. However, the biological mechanism of cocaine's effect on HIV outcomes remains largely unknown. Some studies have suggested that cocaine use may worsen HIV outcomes due to poor adherence to antiretroviral therapy (ART) among HIV-positive participants [2, 12]. Other studies have demonstrated that cocaine's adverse effect on HIV outcomes is independent of ART [10, 11, 13-15], supporting the hypothesis that cocaine exposure may lead to long-lasting pathophysiological changes in the immune system that worsen HIV outcomes.

DNA methylation (DNAm) is an important mechanism associated with many environmental exposures such as smoking, alcohol, and drug misuse [16-26] and diseases such as cancer, diabetes, and cardiovascular diseases [27-32]. Our previous study showed that two DNAm CpG sites in NLRC5 were differentially methylated between HIV-positive and HIV-negative participants in peripheral blood [33]. DNAm may play an important mediation role linking environmental exposure and disease outcomes [34-40]. Environmental exposure such as substance use or toxicants can directly or indirectly affect DNA methylatransferases, causing global or site-specific DNAm changes that may lead to disease [41]. A recent study reported that DNAm sites in PIM3 (energy metabolism) and ABCG1 (lipid metabolism) mediated the association between prenatal famine exposure and long-term metabolic outcomes [38]. Another study reported the mediation effect of cg05575921 (AHRR) between smoking and the risk of bladder cancer among postmenopausal women [42].

Previous studies have shown that the use of cocaine enhances HIV-1 replication and undermines immune function by dysregulating gene expression on HIV-1 entry coreceptors, enhancing HIV-1 cellular toxicity, and dysregulating interleukins (IL) in the host [43, 44]. Cocaine use increases the release of cytokines in immune cells and alters cytokine profile in HIV-infected individuals $[45,46]$. Specifically, cocaine use was positively associated with IL-4 and IL-10 [47], which likely worsens HIV severity and disease progression. Epigenetic mechanisms may play a role in cocaine's effect on the HIV severity because cocaine exposure has been showed to increase the expression of Methyl CpG binding protein 2 (MeCP2) expression [48] as well as DNMT3A and DNMT3B expressions [49] in the animal brains. In a well-matched, case-control human pilot study, cocaine use alters DNA methylation profile in blood [50]. It is plausible that cocaine use may lead to DNAm changes in immune response genes and gene expression changes in cytokine gene family, which further affects HIV progression. Thus, we hypothesized that DNAm may mediate the effect of cocaine exposure on HIV severity.

In this study, we first validated previous findings by examining cocaine's adverse effect on HIV severity and mortality. We further conducted mediation analyses to assess the mediation role of DNAm sites (or CpGs) on cocaine's effect on HIV severity using the Veteran Aging Cohort Study Biomarker Cohort (VACS-BC, $n=1435$ ). To assess how sensitive our results are to the violation of model assumptions and validate our findings using a different approach, we performed the sensitivity analysis [51] and the two-step epigenetic Mendelian randomization (MR) analysis that used genetic variants as instrumental variables to assess the mediation role of DNAm between cocaine use and HIV severity [52]. Our results provide new insights for the role of DNAm on how cocaine affects HIV severity.

\section{Methods}

Study samples

VACS is a prospective cohort study of veterans designed to study substance use and HIV-related outcomes with patient surveys, electronic medical records, and biospecimen data [53]. A baseline survey was conducted at enrollment [53]. The follow-up survey of 5 visits occurred at approximately 1-year intervals [53]. Blood samples were collected in the middle of follow-up for a subset of participants in the cohort (VACS-BC) [54]. A total of 1435 HIV-positive participants from the VACS-BC were used to examine cocaine's effect on mortality and HIV severity, and a subset of participants $(n=875)$ with DNAm data available were used for mediation analyses (Fig. 1). Demographic and clinical information of baseline samples and a subset of the samples at the time of blood collection are summarized in Table 1.

\section{Assessment of cocaine use}

The timeline of cocaine use assessment for each analysis is illustrated in Fig. 1. Information on cocaine use status was self-reported through telephone interviews for a total of 5 visits. We defined the "persistent cocaine use" group as self-reported cocaine use across all 5 visits and the "no cocaine use" group as self-reported no cocaine use across all 5 visits. This definition led to a subset of samples with 265 persistent cocaine users and 202 nonusers for the mediation analyses to eliminate the inconsistent response across 5 visits and examine the effect of long-term cocaine exposure on DNAm and HIV severity.

The frequency of cocaine use was also assessed at baseline (Fig. 1). Each participant was asked "how often 


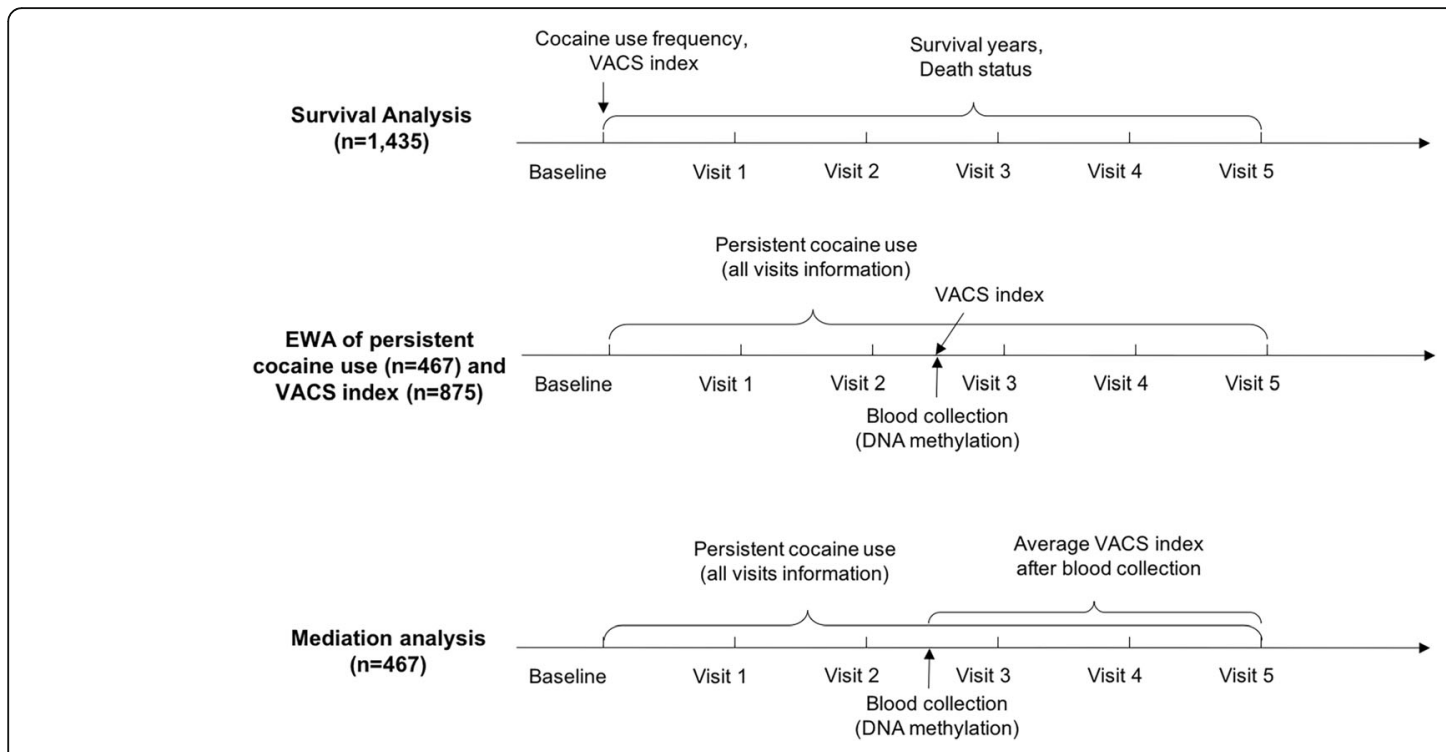

Fig. 1 Timeline of data and blood sample collection for each analysis

Table 1 Sample characteristics in HIV-positive participants

\begin{tabular}{|c|c|c|}
\hline & Baseline $^{a}$ & Follow-up (time of blood collection) \\
\hline Sample size & 1435 & 875 \\
\hline Age [mean (sd)] & $48.8(8.1)$ & $51.5(7.7)$ \\
\hline \multicolumn{3}{|l|}{ Sex } \\
\hline Male & $1399(97.5 \%)$ & $861(98.4 \%)$ \\
\hline Female & $36(2.5 \%)$ & $14(1.6 \%)$ \\
\hline \multicolumn{3}{|l|}{ Race } \\
\hline Caucasian & $290(20.2 \%)$ & $84(9.6 \%)$ \\
\hline African American & $964(67.2 \%)$ & $726(83.0 \%)$ \\
\hline Others & $181(12.6 \%)$ & $65(7.4 \%)$ \\
\hline \multicolumn{3}{|l|}{ Persistent cocaine use status } \\
\hline Persistent cocaine use & & $265(30.3 \%)$ \\
\hline Non-cocaine use & & $202(23.1 \%)$ \\
\hline \multicolumn{3}{|l|}{ Cocaine use at baseline (\%) } \\
\hline Have never tried & $555(38.7 \%)$ & \\
\hline No use in the last year & $540(37.6 \%)$ & \\
\hline Less than once a month & $122(8.5 \%)$ & \\
\hline 1-3 times a month & $103(7.2 \%)$ & \\
\hline 1-3 times a week & $39(2.7 \%)$ & \\
\hline$\geq 4$ times a week & $76(5.3 \%)$ & \\
\hline Cigarette smoking & $762(53.1 \%)$ & $521(59.5 \%)$ \\
\hline VACS index & $30.0(19.1)$ & \\
\hline Average VACS index after blood collection [mean (sd)] & & $39.20(22.4)$ \\
\hline CD4+ count [mean $(\mathbf{s d})]$ & $418.7(273.0)$ & $440.02(289.1)$ \\
\hline $\log 10$ viral load [mean (sd)] & $3.1(1.2)$ & $2.74(1.2)$ \\
\hline Antiviral medication adherence (\%) & $1117(77.8 \%)$ & $666(76.1 \%)$ \\
\hline
\end{tabular}

VACS index: Veteran Aging Cohort Study index

${ }^{a}$ Samples at baseline for the survival analysis

${ }^{\mathrm{b}} \mathrm{A}$ subset of samples at the time of blood collection with DNA methylation measurements 
in the past year have you used cocaine or crack?", from which cocaine frequency of use was coded as an ordinal variable as follows: $0=$ never tried, $1=$ no use in the last year, $2=$ less than once a month, $3=1-3$ times a month, $4=1-3$ times a week, and $5=4$ or more times a week.

\section{Assessment of mortality and HIV severity}

The timeline of HIV severity measurement and survival information for each analysis is shown in Fig. 1. Mortality and survival year information were based on medical records. The VACS index was used as a measure of HIV severity [55-58] and was obtained at each visit and at the time of blood collection (Fig. 1). The VACS index was calculated by summing preassigned points for age, routinely monitored indicators of HIV disease (CD4 count and HIV-1 RNA), and other general indicators of organ system injury [55]. A high VACS index corresponds to worsened HIV outcomes, and the VACS index is positively associated with increased mortality [59]. The VACS index and DNAm profiling were measured at the same time for the selection of candidate mediator CpGs, and the average VACS index after blood collection was used for mediation analyses (Fig. 1).

\section{DNA methylation profiling and quality control}

DNA samples were extracted from blood for a subset of 875 HIV-positive participants (Fig. 1). DNAm was profiled using two different methylation arrays, with 475 samples profiled by the Infinium Human Methylation 450K BeadChip (HM450K, Illumina Inc., CA, USA) and 400 samples later profiled by the Infinium Human Methylation EPIC BeadChip (EPIC, Illumina Inc., CA, USA) [54]. DNA samples were randomly selected for each methylation array regardless of cocaine use status or other clinical demographic variables.

The quality control (QC) for samples measured by each array was conducted separately using the same pipeline as previously described [60] by the $R$ package minfi [61]. After QC, a total of 408,583 CpGs measured by both the HM450k and EPIC array remained for analysis. Six cell-type proportions (CD4+ T cells, CD8+ T cells, NK T cells, B cells, monocytes, and granulocytes) were estimated for each participant using the established method [62]. Negative control probes were designed to capture background signals in Illumina arrays, and negative control principal components (PCs) were extracted by minfi to control for background noise [61]. Batch effect removal was conducted by combat after QC [63].

\section{Genotyping and quality control}

The 1177 samples were genotyped using the Illumina HumanOmniExpress Beadchip and imputed for 18,960, 156 single nucleotide polymorphisms (SNPs). IMUPTE2 (ver 2.3.2) was used for imputation with the reference of 1000 genome phase 3 [64]. QC was conducted using plink (ver 1.90b21) [65]. SNPs and samples with low call rate less than 0.05 were removed. The Hardy-Weinberg equilibrium test cutoff was set to $1 \mathrm{E}-06$. SNPs with minor allele frequency less than 0.01 were filtered.

\section{Statistical analysis}

Cocaine survival analysis among HIV-positive participants at baseline

Survival analysis was conducted using baseline information among 1435 HIV-positive participants with cocaine use frequency (0-5) and other covariates (Fig. 1). Kaplan-Meier analyses on 10-year follow-up among HIV-positive and HIV-negative participants by cocaine use frequency $(0-5)$ at baseline were conducted, and the Kaplan-Meier curves were plotted by using the $\mathrm{R}$ package survminer [66]. A test on ordered differences of Kaplan-Meier curves by cocaine use frequency was conducted by survminer [66].

To adjust for confounding factors, a Cox proportional hazards model was used to assess the hazard ratio of baseline cocaine use frequency $(0-5)$ on mortality during the follow-up using the R package survival [67]. The following model was used to calculate the adjusted hazard ratio among HIV-positive participants:

$$
\begin{aligned}
h(t) & =h_{0}(t) \exp \left(\beta_{1} \text { cocaine use frequency }+\beta_{2}\right. \text { sex } \\
& +\beta_{3} \text { baseline age }+\beta_{4} \text { race }+\beta_{5} \log _{10}(\text { viral load }) \\
& \left.+\beta_{6} C D 4 \text { count }+\beta_{7} \text { antiviral medication adherence }\right)
\end{aligned}
$$

\section{Association between cocaine use frequency and HIV} severity among HIV-positive participants at baseline This analysis was conducted using baseline information on cocaine use frequency (0-5), the VACS index, and other covariates (Fig. 1). The following linear regression model was performed to test the association of cocaine use frequency and HIV severity, adjusting for confounders as shown in the following model:

$$
\begin{aligned}
\text { HIV disease severity } & =\beta_{1} \text { cocaine use frequency } \\
& +\beta_{2} \text { sex }+\beta_{3} \text { age }+\beta_{4} \text { race } \\
& +\beta_{5} \log _{10}(\text { viral load })+\beta_{6} C D 4 \text { count } \\
& +\beta_{7} \text { antiviral medication adherence }
\end{aligned}
$$

\section{Selection of candidate $\mathrm{CpGs}$ by epigenome-wide association (EWA) of persistent cocaine use and HIV severity}

To select candidate CpGs for mediation analysis, we conducted two separate EWAs, one for persistent cocaine use and the other for HIV severity (Fig. 1). Each EWA model adjusted for sex, baseline age, race, smoking, self-reported antiviral medication adherence, white blood cell count, estimated cell-type proportions, and negative control PCs. We used the linear regression 
model with methylation as dependent variable for EWA as described previously [33, 60, 68]. Since CD4+ T cell count is one component of the VACS index, to avoid overrepresented CpGs associated with CD4+ T cells in the EWA results, we extracted the top $1000 \mathrm{CD} 4+\mathrm{T}$ cell-type relevant CpGs based on data from FlowSorted.Blood.450k [69]. The top 2 PCs that in total account for $>80 \%$ variation of the $1000 \mathrm{CD} 4+\mathrm{T}$ cell CpGs were used as covariates in the VACS index EWA model. CpGs with $p<0.001$ in both EWAs for persistent cocaine use and HIV severity were selected as candidate CpGs for mediation analyses. A liberal selection threshold was arbitrarily set to make sure there would be a sufficient number of candidate CpGs for the mediation analysis. To limit confounding by use of other substances, we tested the association of each candidate CpG site with alcohol use, cannabis use, and opioid use based on self-reported data. Alcohol use was assessed by using 3 items of Alcohol Use Diagnosis Identification Testconsumption (AUDIT-C). Cannabis and opioid uses were assessed by asking the same questions as for cocaine use, described earlier.

\section{Single-site mediation analysis and joint mediation analysis}

The selected candidate CpGs were assessed as potential mediators of the association between persistent cocaine use and HIV severity among HIV-positive participants $(n=467)$. We performed single-site mediation analysis using the mediation method as previously described [51] and the $\mathrm{R}$ package mediation [70]. Here, we used the average VACS index after DNAm profiling to ensure the temporality of our mediation hypothesis that DNAm measurement preceded the HIV severity measurement. In our mediation model, we adjusted for sex, age, race, smoking, self-reported antiviral medication adherence, white blood cell count, and estimated cell-type proportions as confounding factors.

We used $M$ to represent the candidate CpGs (mediator), $X$ to represent persistent cocaine use status (exposure), $Y$ to represent the average VACS index after blood collection (outcome), and $C_{i}$ to represent $k$ confounding variables (sex, age, race, smoking, self-report antiviral medication adherence, white blood cell count, estimated CD8 T cells, granulocytes, NK cells, B cells, and monocytes). The mediator model $f(M \mid X, C)$ examined the association between persistent cocaine use and CpGs:

$$
f(M \mid X, C)=\beta_{0} X+\sum_{i=1}^{k} \beta_{i} C_{i}
$$

The outcome model $f(Y \mid X, M, C)$ examined both the direct effect of persistent cocaine use on VACS index and the mediation effect by CpG:

$$
f(Y \mid X, M, C)=\alpha_{0} X+\alpha_{1} M+\sum_{i=1}^{k} \alpha_{i+1} C_{i}
$$

Thus, the mediation effect, or the average causal mediation effect (ACME) of CpG $M$, was $\alpha_{1} \beta_{0}$, the total effect was $\alpha_{0}+\alpha_{1} \beta_{0}$, and the proportion mediated was $\alpha_{1} \beta_{0} /$ $\left(\alpha_{0}+\alpha_{1} \beta_{0}\right)$. The confidence interval and $p$ value were estimated by bootstrapping 1,000,000 iterations.

To assess the robustness of the results if the sequential ignorability assumption was violated, we conducted a sensitivity analysis developed by Imai et al. [51] using the $\mathrm{R}$ package mediation [70]. Sequential ignorability consists of two assumptions: (a) conditional on the covariates $C_{i}$, the exposure $X$ was independent of all potential values of the outcome $Y$ and mediator $M$; and (b) the observed mediator $M$ was independent of all potential outcomes $Y$ given the observed exposure $X$ and covariates $C_{i}$. The sensitivity parameter $\rho$ was calculated on a grid of 0.05 and the $\rho$ at which ACME $=0$ was calculated. For each mediator, sensitivity plots were illustrated to show the estimated ACME and their 95\% confidence interval as a function of $\rho$ (Figure S2). If the $\rho$ at which $\mathrm{ACME}=0$ was close to 0 , it indicates that the mediation analysis was sensitive to violation of the sequential ignorability assumption.

The joint mediation analysis of all significant mediator CpGs was conducted as previously described [71]. The mediator model $f\left(M_{j} \mid X, C\right)$ for multiple mediators $M_{j}\left(M_{1}, M_{2}, \ldots, M_{n}\right)$ was:

$$
f\left(M_{j} \mid X, C\right)=\beta_{0 j} X+\sum_{i=1}^{k} \beta_{i j} C_{i}
$$

The outcome model $f\left(Y \mid X, M_{1}, \ldots, M_{n}, C\right)$ was:

$$
f\left(Y \mid X, M_{1}, \ldots, M_{n}, C\right)=\alpha_{0} X+\sum_{j=1}^{n} \alpha_{j} M_{j}+\sum_{i=1}^{k} \alpha_{i+n} C_{i}
$$

The joint mediation effect of CpGs $M_{1}, \ldots, M_{j}$ is $\sum_{j=1}^{n}$ $\alpha_{j} \beta_{0 j}$, the total effect is $\alpha_{0}+\sum_{j=1}^{n} \alpha_{j} \beta_{0 j}$, and the proportion mediated is $\sum_{j=1}^{n} \alpha_{j} \beta_{0 j} /\left(\alpha_{0}+\sum_{j=1}^{n} \alpha_{j} \beta_{0 j}\right)$. The confidence interval and $p$ value were estimated by bootstrapping 1,000,000 iterations.

\section{Two-step epigenetic Mendelian randomization of cocaine} and HIV severity

To evaluate whether the results from the mediation analysis were influenced by reverse causation or unmeasured confounding, we conducted a two-step epigenetic MR analysis [52] $(n=1177)$ on cocaine use, candidate mediator CpGs, and HIV severity using the inversevariance weighted (IVW) method by $\mathrm{R}$ package MendelianRandomization [72]. 
In step 1, we conducted a two-sample $M R$ on the effect of cocaine use on candidate CpGs $(n=1177)$. Based on a recent meta-analysis of a cocaine dependence genome-wide association study (GWAS) [73], 8 SNPs genotyped in our samples pruned at linkage disequilibrium (LD) $r^{2}<0.1$ by the $\mathrm{R}$ package LDlinkR [74] were used as instrumental variables $(p<1 \mathrm{E}-05)$ (Table S4). We tested the associations between the 8 SNPs and the candidate CpGs, adjusting for age, sex, race, and 5 ancestry PCs using a linear regression model in our sample $(n=1177)$. Based on these summary statistics, we conducted MR using the IVW method to evaluate the effect of cocaine use on candidate CpGs.

In step 2, we conducted a one-sample MR on the effect of candidate CpGs on HIV severity $(n=1177)$. Here, cis-methylation quantitative trait loci (meQTLs) were used as instrumental variables. cis-meQTLs were defined by the distance between a candidate $\mathrm{CpG}$ and a SNP within $1 \mathrm{Mb}$. A linear regression analysis was performed to identify cis-meQTLs, adjusted for age, sex, race, and 5 ancestry PCs. For each candidate CpG, cis-meQTLs with $p<0.01$ after pruning (LD $r^{2}<0.1$ using 1000 genome African ancestry samples as references [75]) were used as instrumental variables in the MR analysis (Table S4) by the $\mathrm{R}$ package LDlinkR [74]. Association between each cis-meQTL and HIV severity was assessed by linear regression, adjusting for age, sex, and 5 ancestry PCs. Similar to the first step, we conducted an MR using the
IVW method to evaluate the effect of candidate CpGs on HIV severity.

\section{Results}

Cocaine use affects HIV severity and mortality among HIV-positive participants

We found that among HIV-positive participants, higher cocaine use frequency was associated with increased mortality ( $p=0.008$, Fig. 2a). This difference was not found among HIV-negative participants ( $p=$ 0.180, Fig. 2b). Using Cox proportional hazards model, this trend remained significant with a hazard ratio (HR) of 1.10 (95\% CI 1.02-1.19, $p=0.011)$, controlling for sex, baseline age, race, viral load, CD4 count, and antiviral medication adherence (Table 2). A higher frequency of cocaine use at baseline was also significantly associated with a higher VACS index (i.e., higher HIV severity, $\beta=1.00, p=0.00027$ ) after adjusting for sex, age, race, viral load, CD4 count, and antiviral medication adherence (Table 2). To account for other drug use, we further adjusted for baseline use of alcohol, cigarette smoking, cannabis, and opioids in the model. Cocaine use frequency remains significantly associated with HIV severity after adjusting for use of other substances $(p=0.049)$. Our results suggest that cocaine use accelerated HIV progression and increased mortality independent of antiviral medication adherence, which is consistent with previous reports [10, 11, 13-15].

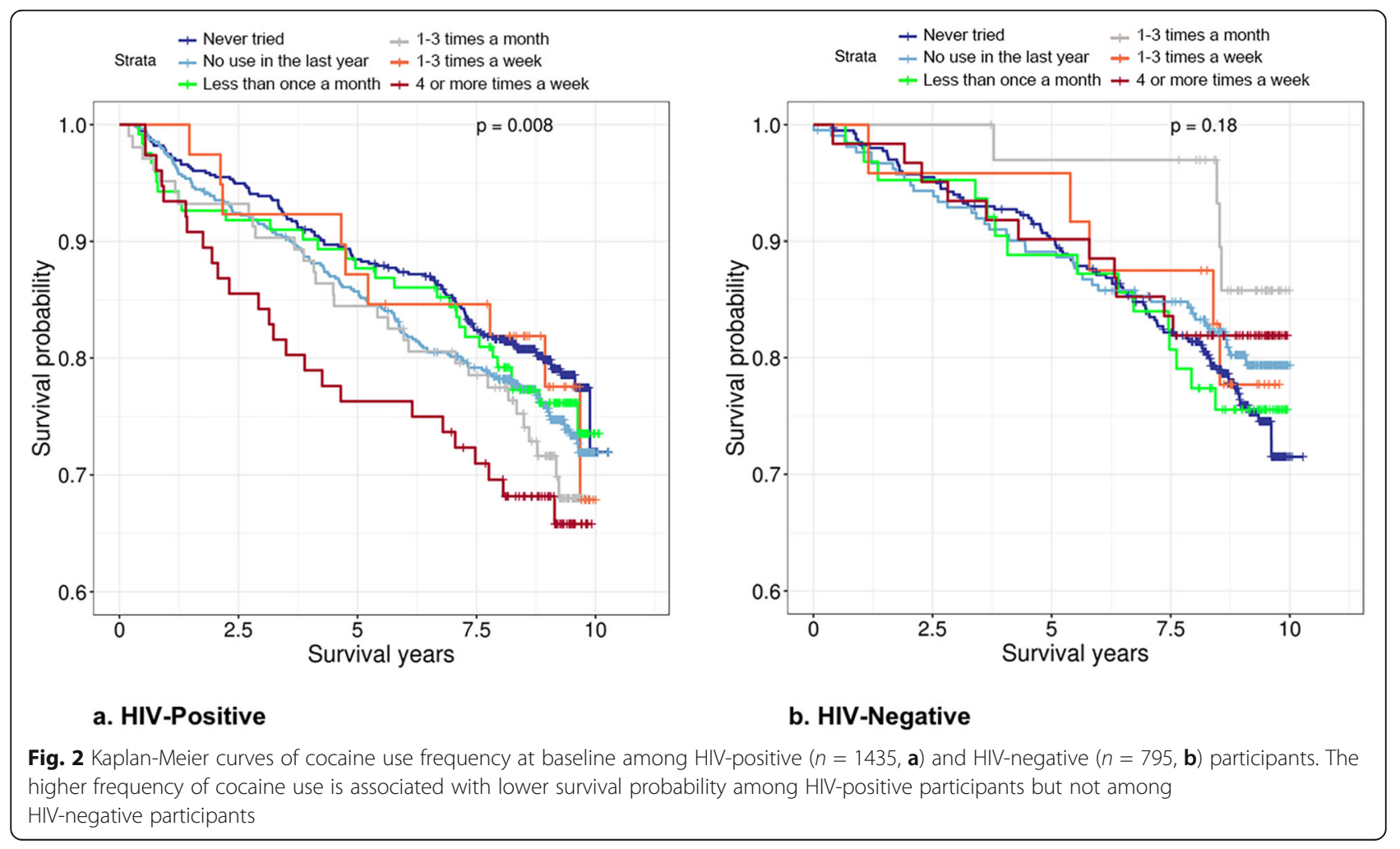


Table 2 Association between cocaine use frequency and HIV severity and survival analysis among HIV-positive participants $(n=1435)$

\begin{tabular}{|c|c|c|c|c|c|c|c|c|}
\hline & \multicolumn{4}{|c|}{ HIV severity $^{a}$} & \multicolumn{4}{|l|}{ Mortality $^{\mathbf{b}}$} \\
\hline & Estimate & SE & $T$ & $p$ value & Hazard ratio & $95 \% \mathrm{Cl}$ & $Z$ & $p$ value \\
\hline Cocaine use frequency ${ }^{c}$ & 1.00 & 0.28 & 3.65 & $2.70 \mathrm{E}-04$ & 1.10 & $(1.02,1.19)$ & 2.54 & $1.10 \mathrm{E}-02$ \\
\hline Sex (reference: male) & 5.78 & 2.33 & 2.48 & $1.34 \mathrm{E}-02$ & 0.27 & $(0.07,1.08)$ & -1.86 & $6.33 \mathrm{E}-02$ \\
\hline $\mathrm{Age}^{\mathrm{c}}$ & 1.09 & 0.05 & 23.58 & $<2 \mathrm{E}-16$ & 1.05 & $(1.04,1.07)$ & 7.35 & $1.90 \mathrm{E}-13$ \\
\hline \multicolumn{9}{|l|}{ Race (reference: Caucasian) } \\
\hline African American & 7.30 & 0.94 & 7.73 & $2.00 \mathrm{E}-14$ & 1.05 & $(0.80,1.39)$ & 0.37 & 7.15E-01 \\
\hline Others & 5.33 & 1.31 & 4.08 & $4.80 \mathrm{E}-05$ & 0.71 & $(0.47,1.09)$ & -1.57 & $1.17 \mathrm{E}-01$ \\
\hline $\log 10$ viral load ${ }^{c}$ & 4.72 & 0.36 & 13.11 & $<2 \mathrm{eE}-16$ & 1.07 & 2 & 1.36 & $1.73 \mathrm{E}-01$ \\
\hline CD4 count ${ }^{c}$ & -0.03 & 0.00 & -19.05 & $<2 \mathrm{E}-16$ & 1.00 & $(1.00,1.00)$ & -3.74 & 1.80E-04 \\
\hline Antiviral medication adherence ${ }^{c}$ & 2.59 & 0.99 & 2.62 & 8.87E-03 & 1.02 & $(0.76,1.37)$ & 0.13 & $8.95 E-01$ \\
\hline
\end{tabular}

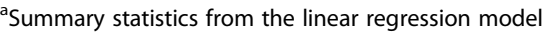

${ }^{b}$ Summary statistics from the Cox proportional hazards model

${ }^{\mathrm{c}}$ Measured at baseline

\section{Selection of candidate DNAm sites for mediation analysis by EWA scan}

The EWA scan of persistent cocaine use showed good control of inflation $(\lambda=1.034$, Figure S1). A total of 497 CpGs met our candidate selection threshold ( $p<0.001)$. The top ranked CpG site, cg22917487, was close to the epigenome-wide significance threshold with a $p$ value of 1.69E-07. This CpG site is located in CX3CR1, a gene that encodes a coreceptor for HIV-1 and leads to rapid HIV progression (Table S1).

The EWA scan of the VACS index also showed good control of inflation $(\lambda=1.116$, Figure S1). There were $876 \mathrm{CpGs}$ that reached the candidate selection threshold $(p<0.001)$ (Table $\mathrm{S} 2)$. Of note, $6 \mathrm{CpGs}$ reached the epigenome-wide significance threshold $(p<1.2 \mathrm{E}-07)$. These CpGs were located near the genes involved in the viral and immune response (PARP9, IFITM1, CD247, IFIT3, VASN, and RUNX1).

We selected candidate CpGs that were both associated with cocaine use and HIV severity $(p<0.001)$ by two separate EWA scans of 408,583 CpGs for mediation analysis. Fourteen CpGs met both candidate selection thresholds. Additionally, cg22917487 in CX3CR1 showed a strong association with cocaine $(p=1.69 \mathrm{E}-07)$ and a marginal association with the VACS index $(p=1.73 \mathrm{E}$ -03). Given its biological plausibility, this CpG was also included as a candidate mediator for mediation analysis. Five of the top 10 VACS index EWA CpGs were selected as candidate mediator CpGs (cg08122652, PARP9, $p=$ 2.30E-10; cg03038262, IFITM1, $p=$ 7.65E-09; cg06188083, IFIT3, $p=4.76 \mathrm{E}-08$; cg08818207, TAP1, $p=2.11 \mathrm{E}-07$; cg26312951, $M X 1, p=2.50 \mathrm{E}-07)$. Overall, a total of 15 CpGs were selected as candidates to assess their potential mediation roles on the association between persistent cocaine use and HIV severity. Notably, the DNAm from each of the 15 CpGs was not associated with cannabis, opioid, or alcohol use $(p>0.05$, Table S3).

\section{Mediation analysis of candidate $\mathrm{CpGs}$ between persistent cocaine use and HIV severity}

We examined the mediation role of DNAm between persistent cocaine use and HIV severity. Twelve out of the 15 candidate $\mathrm{CpGs}$ showed significant mediation effects on the association between persistent cocaine use and the VACS index, with $p$ values ranging from $1.00 \mathrm{E}$ -06 to 0.003 (Table 4). These results remained significant after Bonferroni correction $(p<0.003)$. Each CpG mediator explained between 11.3 and $29.5 \%$ of persistent cocaine use affecting HIV severity. Notably, the direction of mediation effects among these 12 mediator CpGs were the same. The average direct effects of cocaine on HIV severity were attenuated from 0.329 to $0.231-0.291$ after adjusting for each mediator CpG. These 12 CpGs collectively mediated $47.2 \%$ of the cocaine's effects on HIV severity by joint mediation analysis.

We also conducted a sensitivity analysis on these 15 candidate CpGs to assess the robustness of our mediation analysis when the sequential ignorability assumption was violated [51]. The absolute sensitivity parameters at which $\mathrm{ACME}=0$ of the 12 significant mediator CpGs were relatively higher $(|\rho| \geq 0.15)$ than 3 nonsignificant CpGs $(|\rho| \leq$ 0.10) (Table 4, Figure S2). Notably, 6 significant mediator CpGs had $|\rho|$ of 0.30 , indicating that these mediation effects were robust even when the assumptions are slightly violated. The sensitivity analysis showed that our mediation results were relatively stable.

Significant mediator CpGs are located near 11 viral and immune response genes: MX1, PARP9, IFIT3, IFIT M1, NLRC5, EPSTI1, PLSCR1, TAP2, TAP1, CX3CR1, and RIN2. Five CpGs are located on 5' gene regulatory regions, $4 \mathrm{CpGs}$ on gene bodies, $2 \mathrm{CpGs}$ on transcription start sites, and $1 \mathrm{CpG}$ on 3' gene regulatory region. Notably, these $12 \mathrm{CpGs}$ were mostly less methylated in the persistent cocaine use group than in the no cocaine use group (Table 3, Fig. 3). Figure 4 illustrates the 
Table 3 The selected candidate CpG sites by epigenome-wide association (EWA) scan on persistent cocaine use $(n=467)$ and HIV severity $(n=875)$

\begin{tabular}{|c|c|c|c|c|c|c|c|c|c|}
\hline CpG & Chr & Position & $\begin{array}{l}\text { Nearest } \\
\text { gene }\end{array}$ & $\begin{array}{l}\text { Cocaine EWA } \\
\text { effect size }\end{array}$ & $\begin{array}{l}\text { Cocaine } \\
\text { EWA } p \text { value }\end{array}$ & $\begin{array}{l}\text { VACS index } \\
\text { EWA effect size }\end{array}$ & $\begin{array}{l}\text { VACS index } \\
\text { EWA } p \text { value }\end{array}$ & $\begin{array}{l}\text { Reference } \\
\text { gene group }\end{array}$ & $\begin{array}{l}\text { Relations to } \\
\text { CpG islands }\end{array}$ \\
\hline cg22917487 & 3 & 39322103 & CX3CR1 & $1.71 \mathrm{E}-02$ & $1.69 \mathrm{E}-07$ & 1.96E-04 & $1.73 \mathrm{E}-03$ & $\begin{array}{l}\text { Body;TSS200;5UTR; } \\
\text { TSS1500 }\end{array}$ & \\
\hline cg07839457 & 16 & 57023022 & $N L R C 5$ & $-5.18 \mathrm{E}-02$ & $3.16 \mathrm{E}-05$ & $-7.08 \mathrm{E}-04$ & $4.02 \mathrm{E}-04$ & TSS1500 & N_Shore \\
\hline cg26312951 & 21 & 42797847 & $M \times 1$ & $-3.93 E-02$ & $3.24 \mathrm{E}-05$ & $-7.80 \mathrm{E}-04$ & $2.50 \mathrm{E}-07$ & TSS200;5UTR & N_Shore \\
\hline cg06188083 & 10 & 91093005 & IFIT3 & $-5.06 \mathrm{E}-02$ & 3.34E-05 & $-9.96 \mathrm{E}-04$ & $4.76 \mathrm{E}-08$ & Body & \\
\hline cg08122652 & 3 & $1.22 \mathrm{E}+08$ & PARPO & $-6.13 E-02$ & $1.10 \mathrm{E}-04$ & $-1.26 \mathrm{E}-03$ & $2.30 \mathrm{E}-10$ & 5UTR;TSS1500 & N_Shore \\
\hline cg22385827 & 2 & $2.11 \mathrm{E}+08$ & C2orf67 & $8.10 \mathrm{E}-03$ & $2.01 \mathrm{E}-04$ & $1.20 \mathrm{E}-04$ & $7.52 \mathrm{E}-04$ & TSS1500 & Island \\
\hline cg22930808 & 3 & $1.22 \mathrm{E}+08$ & PARPG & $-6.52 E-02$ & $2.44 \mathrm{E}-04$ & $-1.17 \mathrm{E}-03$ & $1.52 \mathrm{E}-06$ & 5UTR;TSS1500 & N_Shore \\
\hline cg06981309 & 3 & $1.46 \mathrm{E}+08$ & $P L S C R 1$ & $-4.29 E-02$ & $2.63 E-04$ & $-5.72 \mathrm{E}-04$ & $4.21 \mathrm{E}-04$ & 5UTR & N_Shore \\
\hline cg03753191 & 13 & 43566902 & EPST/1 & $-1.36 \mathrm{E}-02$ & $2.81 \mathrm{E}-04$ & $-2.44 \mathrm{E}-04$ & $5.50 \mathrm{E}-05$ & TSS1500 & S_Shore \\
\hline cg00096307 & 2 & $1.07 \mathrm{E}+08$ & UXS1 & $-1.12 \mathrm{E}-02$ & $3.59 \mathrm{E}-04$ & $1.74 \mathrm{E}-04$ & $5.73 \mathrm{E}-04$ & Body & \\
\hline cg26396492 & 20 & 19915762 & RIN2 & $-1.26 \mathrm{E}-02$ & 4.76E-04 & $-2.00 \mathrm{E}-04$ & $9.15 E-04$ & Body & \\
\hline cg22940798 & 6 & 32805554 & TAP2 & $-1.93 \mathrm{E}-02$ & $4.82 \mathrm{E}-04$ & $-2.89 \mathrm{E}-04$ & $4.72 \mathrm{E}-04$ & Body & N_Shore \\
\hline cg08623256 & 5 & 3858275 & & $-1.73 \mathrm{E}-02$ & $6.96 \mathrm{E}-04$ & $3.17 \mathrm{E}-04$ & $7.18 \mathrm{E}-04$ & & S_Shelf \\
\hline cg08818207 & 6 & 32820355 & TAP1 & $-2.67 \mathrm{E}-02$ & $8.18 \mathrm{E}-04$ & $-6.18 \mathrm{E}-04$ & $2.11 \mathrm{E}-07$ & Body & N_Shore \\
\hline cg03038262 & 11 & 315262 & IFITM1 & $-2.98 \mathrm{E}-02$ & $9.59 \mathrm{E}-04$ & $-6.46 \mathrm{E}-04$ & $7.65 \mathrm{E}-09$ & 3UTR & N_Shore \\
\hline
\end{tabular}

VACS index Veteran Aging Cohort Study index

mediation effect of cg26312951 (MX1), cg08122652 (PARP9), cg07839457 (NLRC5), and cg22917487 (CX3CR1) on persistent cocaine use affecting HIV severity.

\section{Two-step epigenetic Mendelian randomization of cocaine} and HIV severity

To validate our mediation results while eliminating unmeasured confounding and reverse causation, we used the two-step epigenetic MR method [52] to test our mediation hypotheses $(n=1177)$ : whether cocaine use has a causal effect on candidate CpGs (step 1) and whether candidate CpGs have causal effects on HIV severity (step 2).

In step 1, we conducted the MR analysis based on summary statistics of a meta-analysis of GWAS on cocaine dependence [73]. The effect estimates of the association between $8 \mathrm{SNP}$ instrumental variables and 15 candidate $\mathrm{CpG}$ sites were obtained in our sample. Our MR analysis showed that cocaine had significant MR estimates $(p<0.05)$ on 4 CpGs (cg03753191, EPST I1; cg06188083, IFIT3; cg26312951, MX1; cg22917487, CX3CR1), as shown in Table 5. Three of these CpGs were also among the top significant mediators in our previous mediation analysis (Table 4).

In step 2, we conducted the MR analysis based on cismeQTLs of the candidate CpGs and their association with HIV severity in our sample. Seven CpGs showed significant MR estimates on HIV severity (Table 5). Of note, 3 significant $\mathrm{CpGs}$ in the $\mathrm{MR}$ analysis in step 1 were also significant in step 2 .
Overall, 3 mediator CpGs discovered by the mediation analysis were validated as significant mediators by two-step epigenetic MR analysis (cg03753191, EPSTI1; cg06188083, IFIT3; cg26312951, MX1). Three CpGs without significant mediation effects in the mediation analysis were also found to be nonsignificant in the two-step MR analysis (cg26396492, RIN2; cg22385827, C2orf67; cg08623256).

\section{Discussion}

Our findings provide evidence that cocaine use worsens HIV severity and increases mortality among HIV-positive participants and that cocaine's adverse effects are partially mediated by DNAm in the blood. We identified $12 \mathrm{CpGs}$ that collectively accounted for a total of $47.2 \%$ of cocaine affecting HIV severity. Three of the 12 mediator CpGs were further validated by a two-step epigenetic MR approach, which provides supporting evidence that our mediation results were not affected by unmeasured confounders or reverse causation. The sensitivity analysis showed that our mediation analyses are relatively robust to slight violation of assumptions. These 12 mediator CpGs offer new insights into the mechanisms of how cocaine use may affect HIV outcomes by DNAm.

Methodological considerations are important for examining the mediation effect of DNAm. It is possible that our mediation analyses could be undermined by violation of model assumptions, reverse causation, and unmeasured confounding. To address these concerns, we performed the sensitivity analysis and two-step epigenetic MR analysis to further evaluate the mediation 


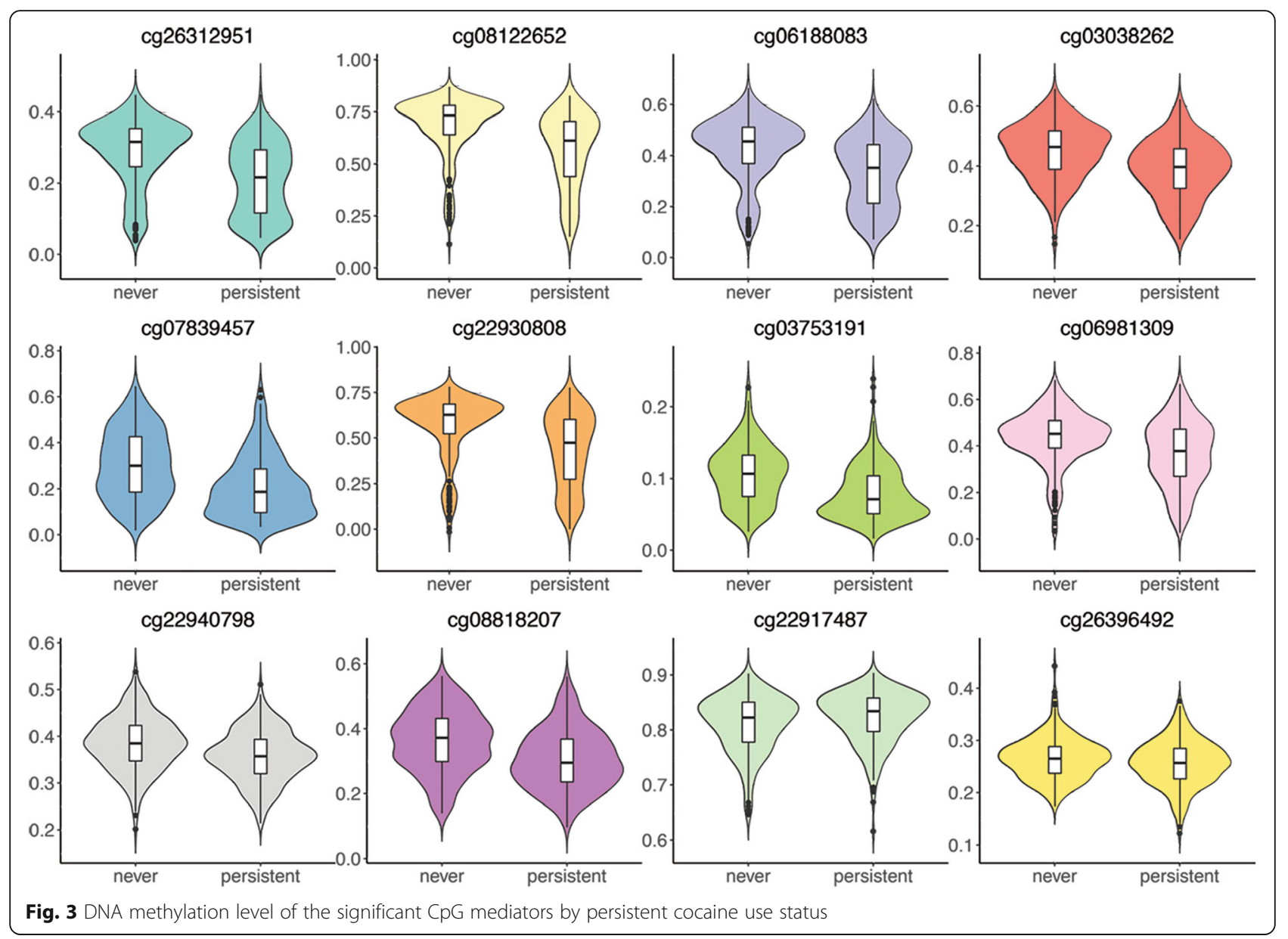

effects of the $12 \mathrm{CpG}$ sites. The results from the sensitivity test showed that the 12 mediator CpGs were robust when slight violation of the sequential ignorability assumption is present. The two-step epigenetic MR analysis confirmed 3 of $12 \mathrm{CpG}$ sites as mediators of cocaine affecting HIV severity and was not affected by reverse causation and unmeasured confounding. Of note, the 8 SNPs used in the MR analysis showed marginal association with cocaine use, which limited their utility as instrumental variables and may explain why 9 CpG sites did not show significant mediation effects in two-step epigenetic MR analysis. In addition, in the mediation analysis, the HIV severity was measured after the blood collection for DNAm profiling to assure that the measurement of mediator precedes the measurement of outcome. Our study design intended to match the temporality of exposure, mediator, and outcome and to avoid reverse causation. Of note, we observed a discrepancy on the direction of cocaine use effect on DNA methylation between EWA scan and step 1 MR analysis. This may happen because EWA scan assessed association while MR evaluated the causal effect by removing reverse causality. This difference might also be due to different ways on adjusting for confounding factors in two models. Additionally, to assess whether cocaine use influenced cell-type proportions as reflected by DNA methylation, we conducted a MR on cocaine affecting six cell-type proportions using the same SNP instruments as used in step $1 \mathrm{MR}$. We found no significant MR estimates across six cell types $(p>0.1)$ (supplementary table S5), suggesting that cocaine use does not directly affect cell-type proportions in our sample. Overall, we took various measures to make sure our mediation results are valid and robust.

We observed that the sum of individual mediation proportion for 12 mediator CpGs exceeded 100\%. An alternative approach is to test the joint mediation effect of all mediators [71]. We found that the 12 mediator CpGs jointly accounted for $47 \%$ of the total effect (effect size = 0.329 ) of cocaine use on HIV severity. This finding indicates that these mediators may affect one another or that there is an interaction effect [71]. For example, several mediator CPG sites are near genes on the response to cytokine pathway (PARP9, PLSCR1, CX3CR1, IFITM1, 


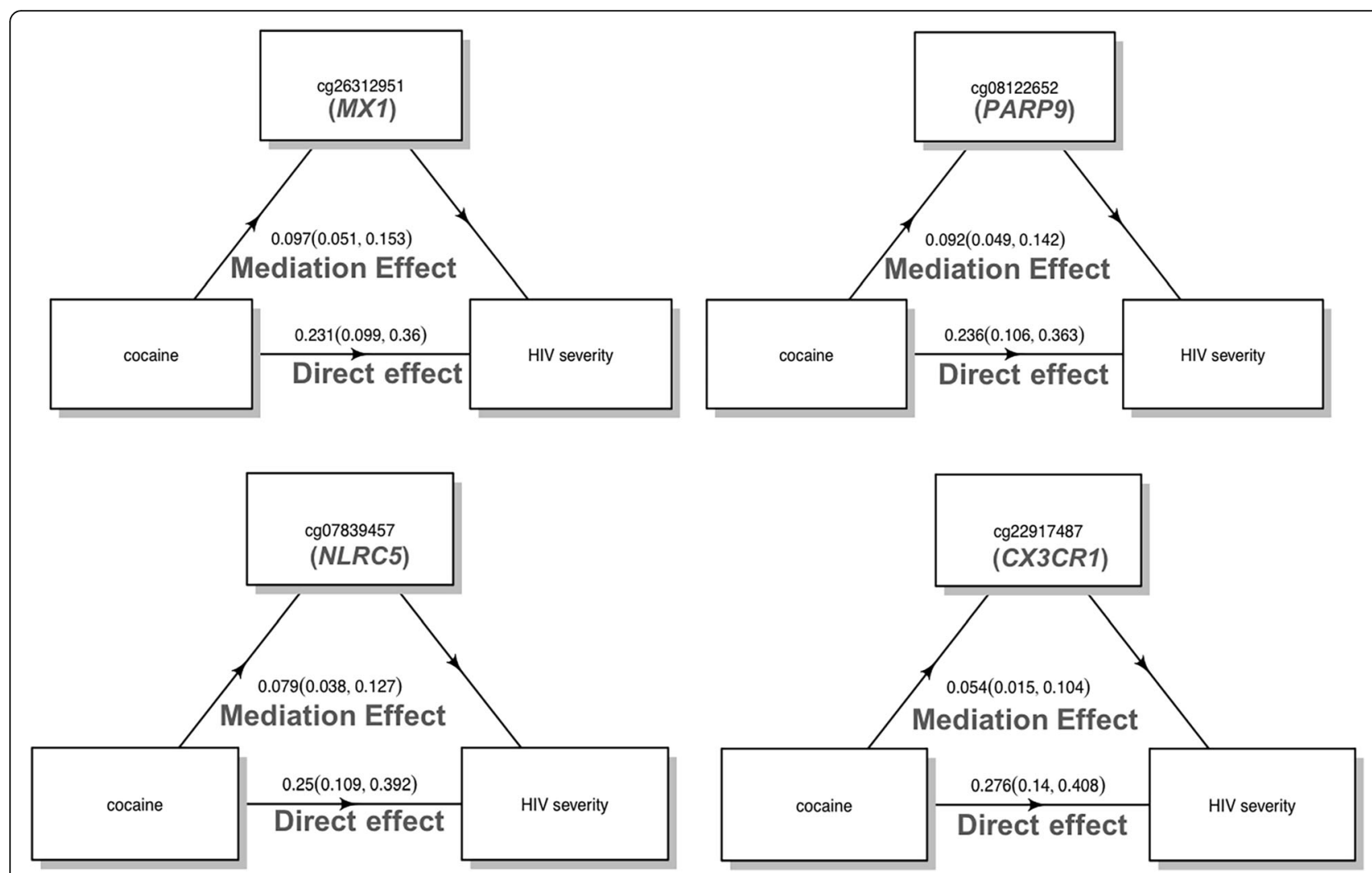

Fig. 4 Significant mediation effect of cg26312951 (MX1), cg08122652(PARP9), cg07839457 (NLRC5), and cg22917487 (CX3CR1) between persistent cocaine use and HIV severity $(p<0.0033)$

IFIT3, MX1, and NLRC5). It is possible that these CpGs may share the common biological pathway on mediating the effect of cocaine on HIV severity.

These 12 CpGs are located in or near 11 biologically meaningful genes that were previously reported to be involved in inflammation, HIV-1 viral replication, and other pathways that play critical roles in HIV progression. Specifically, $\operatorname{cg} 06188083$ on IFIT3 mediated $28.8 \%$ of the variation, and IFIT3 encodes an IFN-induced antiviral protein which acts as an inhibitor of viral processes and viral replication [76]. Another significant mediator CpG site, cg06188083, is located near interferon gene IFITM1. We previously reported the hypomethylation of cg07839457 due to HIV infection, which is located in the promoter region of NLRC5 [33]. This CpG site was also a significant mediator between cocaine and HIV severity in this study. NLRC5 plays an important role in the cytokine response and antiviral immunity through its inhibition of NF-kappa$B$ activation and negative regulation of type I interferon signaling pathways [77]. The converging evidence on cg07839457 (NLRC5) warrants further investigation of its role in HIV infection and progression. Another interesting CpG site, cg22917487 on CX3CR1, showed both a strong association with persistent cocaine use and a significant mediation effect of cocaine affecting HIV severity. CX3CR1 is involved in leukocyte adhesion and migration and was recently identified as an HIV-1 coreceptor [78]. Some studies also showed that genetic variants on CX3CR1 were associated with HIV susceptibility and rapid HIV progression to AIDS [79]. cg25114611, located in the promoter region of $F K B P 5$, is also biologically plausible, given the implication for chronic cocaine administration upregulating FKBP5 expression in rats [80].

Cocaine use commonly cooccurred with the use of other substances, and this may confound cocaine's effects on HIV severity and the mediation effects of CpGs between cocaine use and HIV outcomes. However, our results show that the association between cocaine use and HIV severity remained significant after accounting for smoking, alcohol, cannabis, and opioid use. Additionally, our cocaine use EWA model adjusted for smoking as a covariate, and the selected candidate CpGs were not associated with alcohol, marijuana, and opioid use (Table S3).

There are several strengths of this study. First, instead of selecting candidate mediator CpGs based on the literature or hypotheses, we applied an unbiased epigenome-wide screening to select CpGs associated with both cocaine use and HIV severity. Second, to limit self-reporting bias of cocaine use, we leveraged longitudinal data in defining persistent cocaine use and no cocaine use. We included 


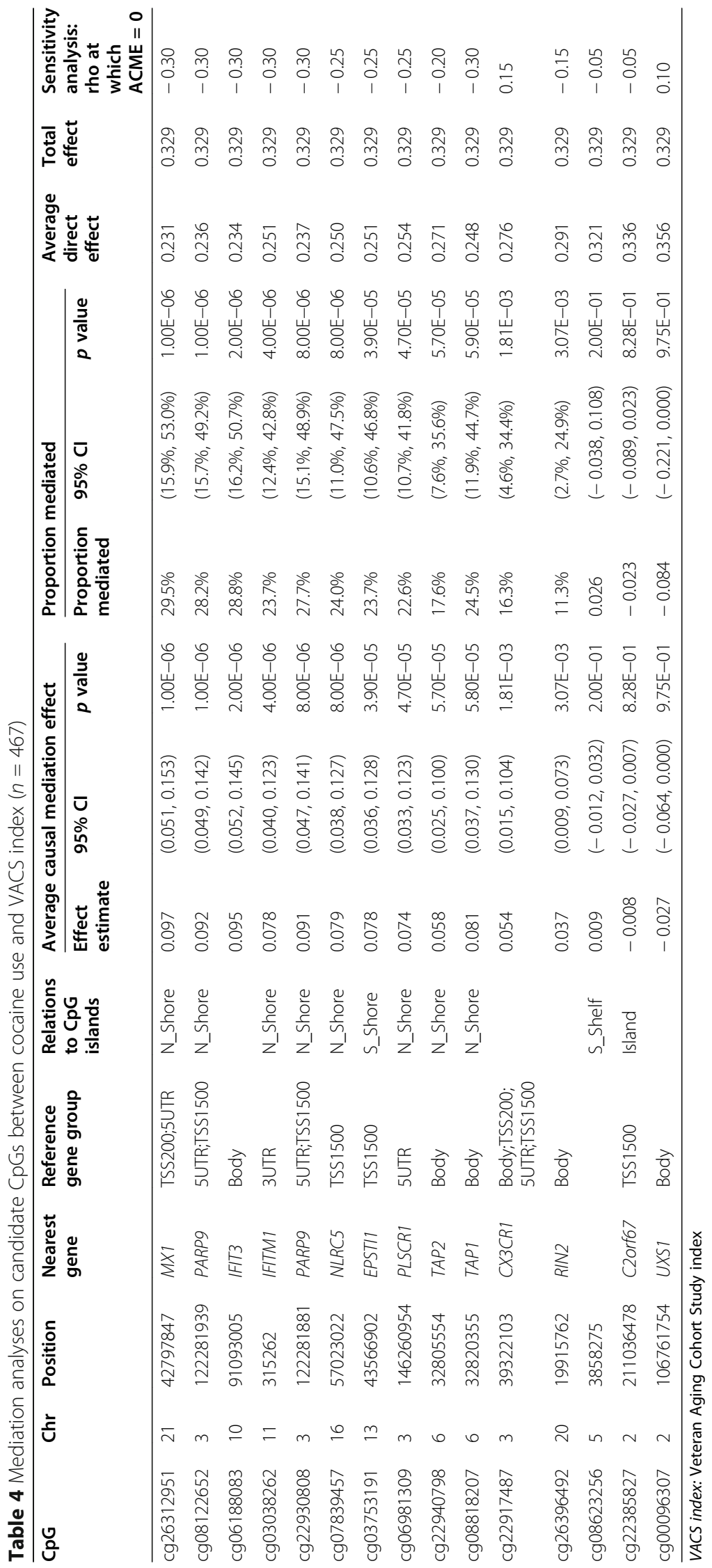




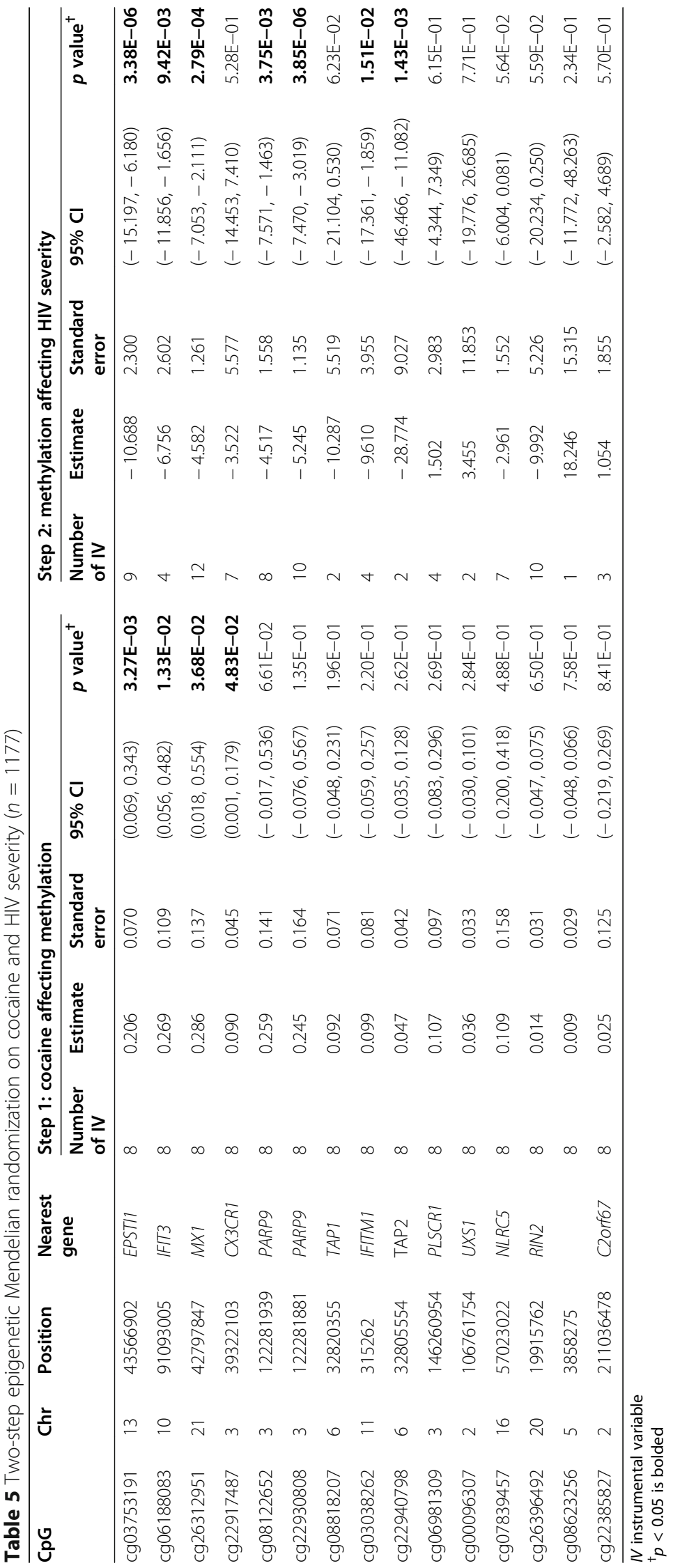


only those participants who consistently reported cocaine use or no cocaine use across all 5 visits for the selection of candidate $\mathrm{CpGs}$ and the mediation analyses. Last, we used the average VACS index after blood collection so that DNAm measurements (mediator) preceded HIV severity (outcome) for the mediation analyses.

One limitation of the study is that our sample size for the mediation analyses is small. However, the strict definition of cocaine use helped reduce self-reporting bias and can potentially increase power by comparing extreme groups. In addition, we used a less stringent criterion when selecting candidate $\mathrm{CpGs}$ for mediation analysis due to the limited sample size to achieve epigenome-wide significance. To our knowledge, there are no sufficiently sized independent cohorts for replication. Although this approach has also been adopted by previous studies [42, 81], using epigenome-wide significant CpG sites as candidate mediators may show stronger signals in the future study with a larger sample size. Additionally, other unmeasured confounding factors such as socioeconomic status may not be fully addressed in the mediation model. Lastly, our samples consisted of mostly male veterans, which may limit the generalizability of our findings.

\section{Conclusions}

We validated previous reports that the use of cocaine worsened HIV severity and increased the risk of all-cause mortality among HIV-positive participants. For the first time, this study found that several biologically meaningful DNAm sites mediated the adverse effect of cocaine use on HIV severity. These results merit future studies to further explore the biological mechanisms revealed by these DNAm sites on how cocaine affects HIV disease outcomes.

\section{Supplementary information}

Supplementary information accompanies this paper at https://doi.org/10. 1186/s13148-020-00934-1.

Additional file 1: Figure S1. Manhattan and quantile-quantile (QQ) plot of persistent cocaine use epigenome-wide association (EWA) $(\lambda=1.034)$ and HIV severity EWA $(\lambda=1.116)$

Additional file 2: Figure S2. The estimated ACME and their 95\% confidence interval as a function of the sensitivity parameter $\rho$ among 15 candidate CpGs.

Additional file 3: Table S1. Epigenome-wide association analysis on persistant cocaine use $(p<0.001)$. Table S2. Epigenome-wide association analysis on HIV severity $(p<0.001)$. Table S3. Epigenome-wide association between candidate CpG sites and other substance use. Table S4. SNP instruments for two-step epigenetic mendelian randomization. Table S5. Mendelian randomization on cocaine use affecting six cell type proportions in blood. Table 6. Gene ontology (GO) pathway enrichment analysis and joint mediation effect.

Additional file 4.

\section{Abbreviations}

ACME: Average causal mediation effect; ART: Antiretroviral therapy; AUDITC: Alcohol Use Diagnosis Identification Test-consumption; Cl: Confidence interval; DNA: Deoxyribonucleic acid; DNAm: DNA methylation;
EWA: Epigenome-wide association; FDR: False discovery rate; GWAS: Genome-wide association study; GO: Gene ontology; HIV: Human immunodeficiency virus; HM450K: Infinium Human Methylation $450 \mathrm{~K}$ BeadChip; EPIC: Infinium Human Methylation EPIC BeadChip; HR: Hazard ratio; IL: Interleukins; IVW: Inverse-variance weighted; LD: Linkage disequilibrium; meQTL: Methylation quantitative trait loci; MR: Mendelian randomization; NK: Natural killer; PC: Principal component; QC: Quality control; VACS: Veterans Aging Cohort Study; VACS-BC: Veteran Aging Cohort Study Biomarker Cohort; VACS index: Veterans Aging Cohort Study index

\section{Acknowledgements}

The authors appreciate the support of the Veteran Aging Study Cohort Biomarker Core and Yale Center of Genomic Analysis.

\section{Authors' contributions}

CS was responsible for data analysis and manuscript preparation. ACJ provided DNA samples and clinical data and contributed to manuscript preparation. XZ was responsible for the bioinformatics data processing. ZW contributed to the mediation analysis and manuscript preparation. $\mathrm{DH}$ and EJ contributed to the analytical approach and the manuscript preparation. KX was responsible for the study design, study protocol, sample preparation, data analysis, interpretation of findings, and manuscript preparation. The author(s) read and approved the final manuscript.

\section{Funding}

The work was supported by the National Institute on Drug Abuse (R03DA039745, R01DA038632, R01DA047063, R01DA047820).

\section{Availability of data and materials}

Demographic and clinical variables and DNAm data for the VACS samples were submitted to the GEO dataset (GSE117861) and are available to the public. All codes for analysis are also available upon a request to the corresponding author.

\section{Ethics approval and consent to participate}

The study was approved by the committee of the Human Research Subject Protection at Yale University and the Institutional Research Board Committee of the Connecticut Veteran Healthcare System. All participants provided written consent.

\section{Competing interests}

All authors declare that they have no conflict of interest.

\section{Author details}

${ }^{1}$ Department of Psychiatry, Yale School of Medicine, New Haven, CT, USA. ${ }^{2}$ Connecticut Veteran Healthcare System, West Haven, CT, USA. ${ }^{3}$ Department of Internal Medicine, Yale School of Medicine, New Haven, CT, USA. ${ }^{4}$ Department of Biostatistics, Yale School of Public Health, New Haven, CT, USA. ${ }^{5}$ GenOmics, Bioinformatics, and Translational Research Center, Biostatistics and Epidemiology Division, RTI International, Research Triangle Park, NC, USA. ${ }^{6}$ Fellow Program, RTI International, Research Triangle Park, NC, USA.

Received: 3 April 2020 Accepted: 3 September 2020

Published online: 14 September 2020

\section{References}

1. Daskalopoulou M, Rodger A, Phillips AN, et al. Recreational drug use, polydrug use, and sexual behaviour in HIV-diagnosed men who have sex with men in the UK: results from the cross-sectional ASTRA study. Lancet HIV. 2014;1(1):e22-31.

2. Cofrancesco J Jr, Scherzer R, Tien PC, et al. Illicit drug use and HIV treatment outcomes in a US cohort. AIDS (London, England). 2008;22(3):357.

3. Hatfield LA, Horvath KJ, Jacoby SM, Simon Rosser BR. Comparison of substance use and risky sexual behavior among a diverse sample of urban, HIV-positive men who have sex with men. J Addict Dis. 2009;28(3):208-18.

4. Grabovac I, Meilinger M, Schalk H, Leichsenring B, Dorner TE. Prevalence and associations of illicit drug and polydrug use in people living with HIV in Vienna. Sci Rep. 2018;8(1):8046.

5. Wolitski RJ, Parsons JT, Gómez CA. Prevention with HIV-seropositive men who have sex with men: lessons from the Seropositive Urban Men's Study 
(SUMS) and the Seropositive Urban Men's Intervention Trial (SUMIT). J Acquir Immune Defic Syndr. 2004;37 Suppl 2:S101-9.

6. Purcell DW, Moss S, Remien RH, Woods WJ, Parsons JT. Illicit substance use, sexual risk, and HIV-positive gay and bisexual men: differences by serostatus of casual partners. Aids. 2005;19 Suppl 1:S37-47.

7. Key substance use and mental health indicators in the United States: results from the 2018 National Survey on Drug Use and Health (HHS Publication No. PEP19-5068, NSDUH Series H-54). Rockville: Center for Behavioral Health Statistics and Quality, Substance Abuse and Mental Health Services Administration; 2019. Retrieved from https://www.samhsa.gov/data/. Accessed 24 Aug 2020.

8. Webber MP, Schoenbaum EE, Gourevitch MN, Buono D, Klein RS. A prospective study of HIV disease progression in female and male drug users. Aids. 1999;13(2):257-62.

9. Vittinghoff E, Hessol NA, Bacchetti P, Fusaro RE, Holmberg SD, Buchbinder SP. Cofactors for HIV disease progression in a cohort of homosexual and bisexual men. J Acquired Immune Deficiency Syndromes (1999). 2001;27(3):308-14.

10. Cook JA, Burke-Miller JK, Cohen MH, et al. Crack cocaine, disease progression, and mortality in a multi-center cohort of HIV-1 positive women. AIDS (London, England). 2008;22(11):1355.

11. Baum MK, Rafie C, Lai S, Sales S, Page B, Campa A. Crack-cocaine use accelerates HIV disease progression in a cohort of HIV-positive drug users. J Acquir Immune Defic Syndr. 2009:50(1):93-9.

12. Sharpe TT, Lee LM, Nakashima AK, Elam-Evans LD, Fleming PL. Crack cocaine use and adherence to antiretroviral treatment among HIV-infected black women. J Community Health. 2004;29(2):117-27.

13. Carrico AW, Johnson MO, Morin SF, et al. Stimulant use is associated with immune activation and depleted tryptophan among HIV-positive persons on anti-retroviral therapy. Brain Behav Immun. 2008;22(8):1257-62.

14. Dash S, Balasubramaniam M, Villalta F, Dash C, Pandhare J. Impact of cocaine abuse on HIV pathogenesis. Front Microbiol. 2015;6:1111.

15. Rasbach DA, Desruisseau AJ, Kipp AM, et al. Active cocaine use is associated with lack of HIV-1 virologic suppression independent of nonadherence to antiretroviral therapy: use of a rapid screening tool during routine clinic visits. AIDS Care. 2013;25(1):109-17.

16. Breitling LP, Yang R, Korn B, Burwinkel B, Brenner H. Tobacco-smokingrelated differential DNA methylation: 27K discovery and replication. Am J Hum Genetics. 2011;88(4):450-7.

17. Joubert BR, Håberg SE, Nilsen RM, et al. 450K epigenome-wide scan identifies differential DNA methylation in newborns related to maternal smoking during pregnancy. Environ Health Perspect. 2012;120(10):1425-31.

18. Lee KW, Pausova Z. Cigarette smoking and DNA methylation. Front Genetics. 2013:4:132.

19. Tsaprouni $L G$, Yang T-P, Bell J, et al. Cigarette smoking reduces DNA methylation levels at multiple genomic loci but the effect is partially reversible upon cessation. Epigenetics. 2014;9(10):1382-96.

20. Gao X, Zhang Y, Saum K-U, Schöttker B, Breitling LP, Brenner H. Tobacco smoking and smoking-related DNA methylation are associated with the development of frailty among older adults. Epigenetics. 2017;12(2):149-56.

21. Zhang $X$, Hu $Y$, Aouizerat $B E$, et al. Machine learning selected smokingassociated DNA methylation signatures that predict HIV prognosis and mortality. Clinical Epigenetic. 2018;10(1):155.

22. Zhang R, Miao Q, Wang C, et al. Genome-wide DNA methylation analysis in alcohol dependence. Addiction Biol. 2013;18(2):392-403.

23. Zhang $\mathrm{H}$, Gelernter J. DNA methylation and alcohol use disorders: progress and challenges. Am J Addictions. 2017;26(5):502-15.

24. Sharp GC, Arathimos R, Reese SE, et al. Maternal alcohol consumption and offspring DNA methylation: findings from six general population-based birth cohorts. Epigenomics. 2018;10(1):27-42.

25. Liu C, Marioni RE, Hedman ÅK, et al. A DNA methylation biomarker of alcohol consumption. Mol Psychiatry. 2018;23(2):422.

26. Joehanes R, Just AC, Marioni RE, et al. Epigenetic signatures of cigarette smoking. Circ Cardiovasc Genet. 2016;9(5):436-47.

27. Feinberg AP, Koldobskiy MA, Göndör A. Epigenetic modulators, modifiers and mediators in cancer aetiology and progression. Nat Rev Genetics. 2016;17:284.

28. Hao X, Luo H, Krawczyk M, et al. DNA methylation markers for diagnosis and prognosis of common cancers. Proceed Natl Acad Sci. 2017;114(28):7414.

29. Michalak EM, Burr ML, Bannister AJ, Dawson MA. The roles of DNA, RNA and histone methylation in ageing and cancer. Nat Rev Mol Cell Biol. 2019. https://doi.org/10.1038/s41580-019-0143-1.
30. Davegårdh C, García-Calzón S, Bacos K, Ling C. DNA methylation in the pathogenesis of type 2 diabetes in humans. Mol Metab. 2018;14:12-25.

31. Zhong J, Agha G, Baccarelli AA. The role of DNA methylation in cardiovascular risk and disease: methodological aspects, study design, and data analysis for epidemiological studies. Circ Res. 2016;118(1):119-31.

32. Nakatochi M, Ichihara S, Yamamoto $\mathrm{K}$, et al. Epigenome-wide association of myocardial infarction with DNA methylation sites at loci related to cardiovascular disease. Clin Epigenetics. 2017;9(1):54.

33. Zhang X, Justice AC, Hu Y, et al. Epigenome-wide differential DNA methylation between HIV-infected and uninfected individuals. Epigenetics. 2016;11(10):750-60.

34. Liu Y, Aryee MJ, Padyukov L, et al. Epigenome-wide association data implicate DNA methylation as an intermediary of genetic risk in rheumatoid arthritis. Nat Biotechnol. 2013;31(2):142.

35. Bind M-A, Lepeule J, Zanobetti A, et al. Air pollution and gene-specific methylation in the Normative Aging Study: association, effect modification, and mediation analysis. Epigenetics. 2014;9(3):448-58.

36. Cao-Lei L, Dancause KN, Elgbeili G, et al. DNA methylation mediates the impact of exposure to prenatal maternal stress on BMI and central adiposity in children at age 131/2years: Project Ice Storm. Epigenetics. 2015;10(8):749-61.

37. Timms JA, Relton CL, Rankin J, Strathdee G, Mckay JA. DNA methylation as a potential mediator of environmental risks in the development of childhood acute lymphoblastic leukemia. Epigenomics. 2016;8(4):519-36.

38. Tobi EW, Slieker RC, Luijk R, et al. DNA methylation as a mediator of the association between prenatal adversity and risk factors for metabolic disease in adulthood. Sci Adv. 2018;4(1):eaao4364.

39. Barker ED, Walton E, Cecil CA. Annual Research Review: DNA methylation as a mediator in the association between risk exposure and child and adolescent psychopathology. J Child Psychol Psychiatry. 2018;59(4):303-22.

40. Rutten BPF, Mill J. Epigenetic mediation of environmental influences in major psychotic disorders. Schizophrenia Bulletin. 2009;35(6):1045-56.

41. Ladd-Acosta C, Fallin MD. The role of epigenetics in genetic and environmental epidemiology. Epigenomics. 2016;8(2):271-83.

42. Jordahl KM, Phipps Al, Randolph TW, et al. Differential DNA methylation in blood as a mediator of the association between cigarette smoking and bladder cancer risk among postmenopausal women. Epigenetics. 2019:1-9. https://doi.org/10.1080/15592294.2019.1631112.

43. Shirazi J, Shah S, Sagar D, et al. Epigenetics, drugs of abuse, and the retroviral promoter. J Neuroimmune Pharmacol. 2013;8(5):1181-96.

44. Dhillon NK, Williams R, Peng F, et al. Cocaine-mediated enhancement of virus replication in macrophages: implications for human immunodeficiency virus-associated dementia. J Neurovirol. 2007;13(6):483-95.

45. Atluri VS, Pilakka-Kanthikeel S, Garcia G, et al. Effect of cocaine on HIV infection and inflammasome gene expression profile in HIV infected macrophages. Sci Rep. 2016;6:27864.

46. Castro FOF, Silva JM, Dorneles GP, et al. Distinct inflammatory profiles in HIV-infected individuals under ART using cannabis, cocaine or cannabis plus cocaine. AIDS. 2019. https://doi.org/10.1097/QAD.0000000000002296.

47. Parikh N, Dampier W, Feng R, et al. Cocaine alters cytokine profiles in HIV-1infected African American individuals in the DrexelMed HIV/AIDS genetic analysis cohort. J Acquir Immune Defic Syndr. 2014;66(3):256-64.

48. Robison AJ, Nestler EJ. Transcriptional and epigenetic mechanisms of addiction. Nat Rev Neurosci. 2011;12(11):623-37.

49. Anier K, Malinovskaja K, Aonurm-Helm A, Zharkovsky A, Kalda A. DNA methylation regulates cocaine-induced behavioral sensitization in mice. Neuropsychopharmacology. 2010;35(12):2450-61.

50. Camilo C, Maschietto M, Vieira HC, et al. Genome-wide DNA methylation profile in the peripheral blood of cocaine and crack dependents. Braz J Psychiatry. 2019;41(6):485-93.

51. Imai K, Keele L, Tingley D. A general approach to causal mediation analysis. Psychol Methods. 2010;15(4):309.

52. Relton CL, Davey Smith G. Two-step epigenetic Mendelian randomization: a strategy for establishing the causal role of epigenetic processes in pathways to disease. Int J Epidemiol. 2012;41 (1):161-76.

53. Justice AC, Dombrowski E, Conigliaro J, et al. Veterans aging cohort study (VACS): overview and description. Medical care. 2006;44(8 Suppl 2):S13.

54. Veterans Aging Cohort Study. VACS biomarker cohort description. (2016).

55. Tate JP, Justice AC, Hughes MD, et al. An internationally generalizable risk index for mortality after one year of antiretroviral therapy. AIDS (London, England). 2013;27(4):563-72. 
56. Justice AC, Modur SP, Tate JP, et al. Predictive accuracy of the Veterans Aging Cohort Study index for mortality with HIV infection: a North American cross cohort analysis. J Acquir Immune Defic Syndro (1999). 2013; 62(2):149-63.

57. Bebu I, Tate J, Rimland D, et al. The VACS index predicts mortality in a young, healthy HIV population starting highly active antiretroviral therapy. J. Acquir. Immune. Defic. Syndr. 2014;65(2):226-30.

58. Brown ST, Tate JP, Kyriakides TC, et al. The VACS index accurately predicts mortality and treatment response among multi-drug resistant HIV infected patients participating in the options in management with antiretrovirals (OPTIMA) study. PLoS One. 2014;9(3):e92606.

59. Justice AC, Modur S, Tate JP, et al. Predictive accuracy of the Veterans Aging Cohort Study (VACS) index for mortality with HIV infection: a north American cross cohort analysis. J Acquir Immune Defic Syndr (1999). 2013; 62(2):149.

60. Lehne B, Drong AW, Loh $M$, et al. A coherent approach for analysis of the Illumina HumanMethylation450 BeadChip improves data quality and performance in epigenome-wide association studies. Genome biology. 2015;16(1):37.

61. Aryee MJ, Jaffe AE, Corrada-Bravo H, et al. Minfi: a flexible and comprehensive Bioconductor package for the analysis of Infinium DNA methylation microarrays. Bioinformatics. 2014;30(10):1363-9.

62. Houseman EA, Accomando WP, Koestler DC, et al. DNA methylation arrays as surrogate measures of cell mixture distribution. BMC Bioinformatics. 2012; 13(1):86.

63. Johnson WE, Li C, Rabinovic A. Adjusting batch effects in microarray expression data using empirical Bayes methods. Biostatistics. 2007:8(1): $118-27$.

64. Howie BN, Donnelly P, Marchini J. A flexible and accurate genotype imputation method for the next generation of genome-wide association studies. PLoS Genet. 2009;5(6):e1000529.

65. Purcell $\mathrm{S}$, Neale $\mathrm{B}$, Todd-Brown $\mathrm{K}$, et al. PLINK: a tool set for whole-genome association and population-based linkage analyses. Am J Hum Genetics. 2007:81(3):559-75

66. Kassambara A, Kosinski M, Biecek P. survminer: drawing survival curves using'ggplot2'. R package version 0.3 1. 2017. https://mi2-warsaw.github.io/ publication/kassambara2017survminer/.

67. Therneau T. A package for survival analysis in S. version 2.38; 2015.

68. Zhang $X, \mathrm{Hu} Y$, Justice AC, et al. DNA methylation signatures of illicit drug injection and hepatitis $C$ are associated with HIV frailty. Nat Commun. 2017; 8(1):2243.

69. Jaffe A. Flowsorted. blood. 450k: illumina humanmethylation data on sorted blood cell populations. R Package Version. 2015;1(0). https://bioconductor. org/packages/release/data/experiment/html/FlowSorted.Blood.450k.html.

70. Tingley D, Yamamoto T, Hirose K, Keele L, Imai K. Mediation: R package for causal mediation analysis; 2014

71. Vanderweele T, Vansteelandt S. Mediation analysis with multiple mediators. Epidemiol Methods. 2014;2(1):95-115.

72. Yavorska OO, Burgess S. MendelianRandomization: an R package for performing Mendelian randomization analyses using summarized data. Int J Epidemiol. 2017:46(6):1734-9.

73. Cabana-Domínguez J, Shivalikanjli A, Fernàndez-Castillo N, Cormand B. Genome-wide association meta-analysis of cocaine dependence: shared genetics with comorbid conditions. Progress in Neuro-Psychopharmacology and Biological Psychiatry. 2019:94:109667.

74. Myers TA, Chanock SJ, Machiela MJ. LDlinkR: an R package for rapidly calculating linkage disequilibrium statistics in diverse populations. Frontiers in Genetics. 2020;11:157.

75. Auton A, Abecasis GR, Altshuler DM, et al. A global reference for human genetic variation. Nature. 2015;526(7571):68-74.

76. Schmeisser H, Mejido J, Balinsky CA, et al. Identification of alpha interferon-induced genes associated with antiviral activity in Daudi cells and characterization of IFIT3 as a novel antiviral gene. J Virol. 2010; 84(20):10671-80.

77. Cui J, Zhu L, Xia X, et al. NLRC5 negatively regulates the NF-kappaB and type I interferon signaling pathways. Cell. 2010;141(3):483-96.

78. Garin A, Tarantino N, Faure S, et al. Two novel fully functional isoforms of CX3CR1 are potent HIV coreceptors. J Immunol. 2003:171(10):5305-12.

79. Faure S, Meyer L, Costagliola D, et al. Rapid progression to AIDS in HIV+ individuals with a structural variant of the chemokine receptor CX3CR1. Science. 2000;287(5461):2274-7.
80. Connelly $\mathrm{KL}$, Unterwald EM. Chronic cocaine administration upregulates FKBP5 in the extended amygdala of male and female rats. Drug Alcohol Depend. 2019;199:101-5.

81. Chu SH, Loucks EB, Kelsey KT, et al. Sex-specific epigenetic mediators between early life social disadvantage and adulthood BMI. Epigenomics. 2018;10(6):707-22.

\section{Publisher's Note}

Springer Nature remains neutral with regard to jurisdictional claims in published maps and institutional affiliations.
Ready to submit your research? Choose BMC and benefit from:

- fast, convenient online submission

- thorough peer review by experienced researchers in your field

- rapid publication on acceptance

- support for research data, including large and complex data types

- gold Open Access which fosters wider collaboration and increased citations

- maximum visibility for your research: over $100 \mathrm{M}$ website views per year

At BMC, research is always in progress.

Learn more biomedcentral.com/submissions 